\title{
ALMA Discovery of Dust Belts around Proxima Centauri
}

DOI:

10.3847/2041-8213/aa978b

\section{Document Version}

Final published version

Link to publication record in Manchester Research Explorer

\section{Citation for published version (APA):}

Anglada, G., Amado, P. J., Ortiz, J. L., Gómez, J. F., Maclas, E., Alberdi, A., Osorio, M., Gómez, J. L., GregorioMonsalvo, I. D., Pérez-Torres, M. A., Anglada-Escudé, G., Berdinas, Z. M., Jenkins, J. S., Jimenez-Serra, I., Lara, L. M., López-González, M. J., López-Puertas, M., Morales, N., Ribas, I., … Rodriguez, E. (2017). ALMA Discovery of Dust Belts around Proxima Centauri. Astrophysical Journal Letters, 850(1), [L6]. https://doi.org/10.3847/20418213/aa978b

\section{Published in:}

Astrophysical Journal Letters

\section{Citing this paper}

Please note that where the full-text provided on Manchester Research Explorer is the Author Accepted Manuscript or Proof version this may differ from the final Published version. If citing, it is advised that you check and use the publisher's definitive version.

\section{General rights}

Copyright and moral rights for the publications made accessible in the Research Explorer are retained by the authors and/or other copyright owners and it is a condition of accessing publications that users recognise and abide by the legal requirements associated with these rights.

\section{Takedown policy}

If you believe that this document breaches copyright please refer to the University of Manchester's Takedown Procedures [http://man.ac.uk/04Y6Bo] or contact uml.scholarlycommunications@manchester.ac.uk providing relevant details, so we can investigate your claim.

\section{OPEN ACCESS}




\title{
ALMA Discovery of Dust Belts around Proxima Centauri
}

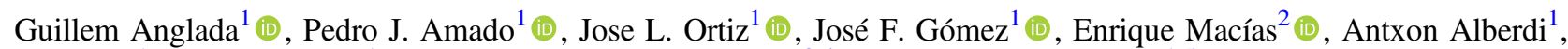 \\ Mayra Osorio ${ }^{1}$, José L. Gómez ${ }^{1}$ (D), Itziar de Gregorio-Monsalvo ${ }^{3,4}$, Miguel A. Pérez-Torres ${ }^{1,5}$ (D), Guillem Anglada-Escudé ${ }^{6}$ (iD), \\ Zaira M. Berdiñas ${ }^{1,7}$, James S. Jenkins ${ }^{7}$ (D), Izaskun Jimenez-Serra ${ }^{6}$ (D), Luisa M. Lara ${ }^{1}$, Maria J. López-González ${ }^{1}$, \\ Manuel López-Puertas ${ }^{1}$ (iD), Nicolas Morales ${ }^{1}$, Ignasi Ribas ${ }^{8}$ (D), Anita M. S. Richards ${ }^{9}$, Cristina Rodríguez-López ${ }^{1}$ (iD, and \\ Eloy Rodriguez ${ }^{1}$ \\ ${ }^{1}$ Instituto de Astrofísica de Andalucía, CSIC, Glorieta de la Astronomía s/n, E-18008 Granada, Spain; guillem@iaa.es \\ ${ }^{2}$ Department of Astronomy, Boston University, 725 Commonwealth Avenue, Boston, MA 02215, USA \\ ${ }^{3}$ European Southern Observatory, Alonso de Córdova 3107, Vitacura, Santiago, Chile \\ ${ }^{4}$ Joint ALMA Observatory, Alonso de Córdova 3107, Vitacura, Casilla 19001, Santiago 19, Chile \\ ${ }^{5}$ Visiting Scientist: Departamento de Fsica Teórica, Facultad de Ciencias, Universidad de Zaragoza, E-50009, Zaragoza, Spain \\ ${ }_{7}^{6}$ School of Physics and Astronomy, Queen Mary University of London, 327 Mile End Road, London E1 4NS, UK \\ ${ }^{7}$ Departamento de Astronomía, Universidad de Chile, Camino del Observatorio, 1515 Las Condes, Santiago, Chile \\ ${ }^{8}$ Institut de Ciències de l'Espai (IEEC-CSIC), C/ Can Magrans s/n, Campus UAB, E-08193 Bellaterra, Spain \\ 9 JBCA, School of Physics and Astronomy, University of Manchester, Manchester M13 9PL, UK \\ Received 2017 October 15; revised 2017 October 29; accepted 2017 November 1; published 2017 November 15
}

\begin{abstract}
Proxima Centauri, the star closest to our Sun, is known to host at least one terrestrial planet candidate in a temperate orbit. Here we report the Atacama Large Millimeter/submillimeter Array (ALMA) detection of the star at $1.3 \mathrm{~mm}$ wavelength and the discovery of a belt of dust orbiting around it at distances ranging between 1 and 4 au, approximately. Given the low luminosity of the Proxima Centauri star, we estimate a characteristic temperature of about $40 \mathrm{~K}$ for this dust, which might constitute the dust component of a small-scale analog to our solar system's Kuiper Belt. The estimated total mass, including dust and bodies up to $50 \mathrm{~km}$ in size, is of the order of 0.01 Earth masses, which is similar to that of the solar Kuiper Belt. Our data also show a hint of warmer dust closer to the star. We also find signs of two additional features that might be associated with the Proxima Centauri system that still require further observations to be confirmed: an outer extremely cold (about $10 \mathrm{~K}$ ) belt around the star at about $30 \mathrm{au}$, with an orbital plane that is tilted about $45^{\circ}$ with respect to the plane of the sky; additionally, we marginally detect a compact $1.3 \mathrm{~mm}$ emission source at a projected distance of about 1.2 arcsec from the star, the nature of which is still unknown.
\end{abstract}

Key words: circumstellar matter - planetary systems - radio continuum: planetary systems - stars: individual (Proxima Centauri)

\section{Introduction}

Cold debris disks around main-sequence stars (e.g., Greaves et al. 2004; di Folco et al. 2007; Lestrade et al. 2012; ChavezDagostino et al. 2016; MacGregor et al. 2016) are leftover planetesimals that could not agglomerate into larger bodies during the process of planet formation. In these disks, dust grains are continuously replenished by dust particles resulting from the grinding-down of larger planetesimals in destructive collisions (the so-called collisional cascade; Wyatt et al. 2007b). These processes produce a second generation of dust grains with a wide size distribution, the thermal emission of which is observable from far-infrared (far-IR) to mm wavelengths (Wyatt 2008; Matthews et al. 2014 and references therein). Dust is usually distributed as a belt within the periphery of the system in a way analogous to the Kuiper Belt in our solar system. While most of the known debris disks present cold dust in narrow belts at tens of au, a small fraction host a hot dust component within a few au, analogous to the Asteroid belt or Zodiacal dust (e.g., Absil et al. 2013; Marino et al. 2017).

The study of the present-day structure and dynamics of these dust belts can provide important information about the formation and evolution of exoplanetary systems, in a way similar to our solar system. For example, it is thought that the present-day solar Kuiper Belt is more extended and 100 times less massive than it was initially. This depletion in mass is explained in terms of dynamical instabilities due to early Jupiter-Saturn interactions (the Nice model; Morbidelli et al. 2005).

Proxima Centauri, at a distance of 1.3 pc (van Leeuwen 2007), is the closest star to the Sun. It is an M5.5V star with $T_{\text {eff }}=$ $3000 \mathrm{~K}, M_{*}=0.12 M_{\odot}, R_{*}=0.15 R_{\odot}$, and $L_{*}=0.0015 L_{\odot}$ (Ribas et al. 2017); it belongs to a triple system (Kervella et al. 2017) and its age is estimated to be $\sim 5$ Gyr (Bazot et al. 2016), if coeval formation is assumed. The discovery, using Doppler data, of a terrestrial planet candidate $\left(m_{p} \sin i=1.3 M_{\oplus}\right)$ orbiting the star at 0.05 au (Anglada-Escudé et al. 2016) has triggered the study of the main features of this stellar-planetary system. Raymond et al. (2011) showed that debris disks are signposts of terrestrial planet formation. Thus, one might expect that Proxima Centauri is associated with a Kuiper Belt analog that would allow us to learn about its planetary system history and architecture. Besides the intrinsic interest of studying a Kuiper Belt analog in Proxima Centauri, imaging this belt would allow us to constrain the inclination angle of the orbital plane of the planet Proxima $b$ and therefore to determine its true mass.

In this Letter we report the first results of ALMA band 6 observations toward the star closest to our Sun, aiming at characterizing the architecture of its planetary system through the thermal emission of the surrounding dust. 


\section{Observations}

We observed Proxima Centauri with the Atacama Large Millimeter/submillimeter Array (ALMA) at $1.3 \mathrm{~mm}$, using both the Atacama Compact Array (ACA) of $7 \mathrm{~m}$ antennas and the main ALMA array of $12 \mathrm{~m}$ antennas. In all of the cases we observed in dual polarization, with four spectral windows centered at 225, 227, 239, and $241 \mathrm{GHz}$, each with a $1.875 \mathrm{GHz}$ effective bandwidth split into 120 channels. ACA observations were made between 2017 January 21 and 2017 March 24, with 8-11 antennas available, in 13 separate sessions of $\sim 1.6 \mathrm{hr}$ each, including overheads. Ganymede, Callisto, Titan, J1427-4206, and J1517-2422 were used for absolute flux calibration, J1427-4206 and J1924-2914 for bandpass calibration, and J1424-6807 and J1329-5608 (within 5.5 and $10^{\circ} .0$ of the target, respectively) for phase calibration. Observations were sensitive to angular scales $\lesssim 29^{\prime \prime}$. At this band, the primary beam FWHM is $\sim 39^{\prime \prime}$ and its first null is at a radius of $\sim 46^{\prime \prime}$. The $12 \mathrm{~m}$ array, with 41 antennas available, was used in a single session of $2.6 \mathrm{hr}$ on 2017 April 25. J1617-5848 and Titan were used for absolute flux calibration, J1427-4206 for bandpass calibration, and J1424-6807 (within 5.5 of the target) for phase calibration. Observations were sensitive to angular scales $\lesssim 6^{\prime \prime}$. The primary beam FWHM is $\sim 23^{\prime \prime}$ and its first null is at a radius of $\sim 27^{\prime \prime}$.

Considering the large proper motions and parallax of Proxima Centauri, the phase center of the observations was updated from scan to scan to track the source position with sub-milliarcsec accuracy. This was done by assuming source coordinates at epoch and equinox $\mathrm{J} 2000.0$ of R.A. $=14^{\mathrm{h}} 29^{\mathrm{m}} 42^{\mathrm{s}} .9485$, decl. $=$ $-62^{\circ} 40^{\prime} 46^{\prime \prime} 163$, proper motions of -3775.75 and 765.54 mas $\mathrm{yr}^{-1}$ (van Leeuwen 2007) in R.A. and decl., respectively, and a parallax of 768.13 mas (Lurie et al. 2014).

Data were processed using the Common Astronomy Software Applications (CASA) package. Calibration was performed with the ALMA pipeline, using CASA versions 4.7.0 and 4.7.2 for ACA and $12 \mathrm{~m}$ array data, respectively. Images were obtained by combining all of the spectral windows in multifrequency synthesis mode, applying a robust parameter of 0.5 , and deconvolving with the CLEAN algorithm.

Self-calibration was attempted, but was unsuccessful due to insufficient signal-to-noise ratio $(\mathrm{S} / \mathrm{N})$. Flux densities and intensities were measured with task IMSTAT. Source positions and sizes were determined with task IMFIT. Positional and flux uncertainties include absolute errors $(5 \%$ of the resolution in astrometry and 7\% in flux calibration) and relative errors due to noise in the images. All of the errors quoted in this Letter are at a $1 \sigma$ level.

Figure 1 shows the $1.3 \mathrm{~mm}$ ACA image (synthesized beam $\left.\mathrm{FWHM} \simeq 6^{\prime \prime}\right)$. As the phase center was changed in each session to track the source position, all of the data were assigned the formal position of the first epoch of observation, prior to combination of all epochs. Our image shows an unresolved source coinciding within the uncertainty $(0,4)$ with the optical position of Proxima Centauri. The source has a flux density of $340 \pm 60 \mu \mathrm{Jy}$.

Figure 2 shows the $1.3 \mathrm{~mm}$ image obtained with the ALMA $12 \mathrm{~m}$ array (synthesized beam FWHM $\simeq 0$."7). The image shows a main central source, the emission peak of which is located at ICRS coordinates R.A. $=14^{\mathrm{h}} 29^{\mathrm{m}} 33^{\mathrm{s}} .445 \pm 0.005$, decl. $=$ $-62^{\circ} 40^{\prime} 33$ !' $40 \pm 0$ "' 03 , coinciding within 0 ." 1 with the optical position of the star at the epoch of observation. The flux density is $106 \pm 12 \mu \mathrm{Jy}$ and the intensity peak is $100 \pm 12 \mu \mathrm{Jy}_{\text {beam }}{ }^{-1}$.

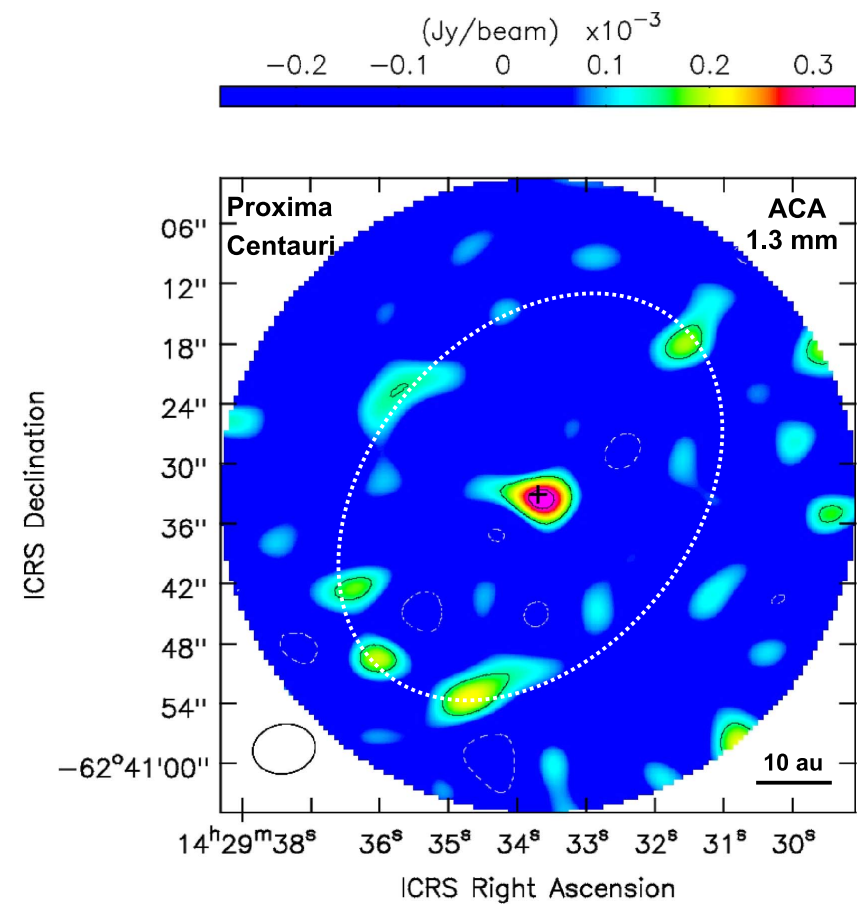

Figure 1. ACA $7 \mathrm{~m}$ array image of the $1.3 \mathrm{~mm}$ emission toward Proxima Centauri. Contours are $-3,3$, and 6 times $50 \mu \mathrm{Jy}$ beam $^{-1}$, the $\mathrm{rms}$ of the image. The position of the star on 2017 January 21, measured from an optical image (error $\simeq 0$." 1 ), is marked with a + sign. The ellipse shows the belt of radius 30 au suggested by the azimuthally averaged intensity profile (see the text). The primary beam response correction has not been applied to this image to better represent the noise distribution. The figure shows the region where the primary beam response is $>20 \%$ of that at the field center. The synthesized beam $\left(6{ }^{\prime \prime} 28 \times 4^{\prime \prime} 96, \mathrm{PA}=-80^{\circ} 4\right)$ is shown in the bottom-left corner.
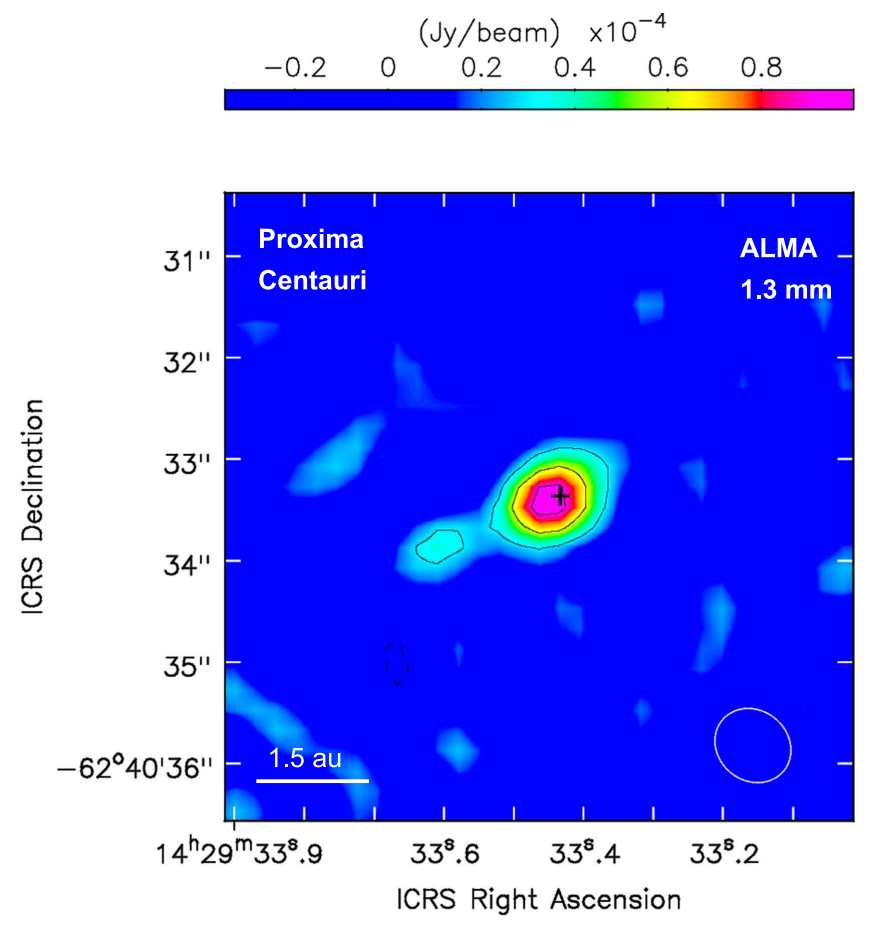

Figure 2. ALMA $12 \mathrm{~m}$ array image of the $1.3 \mathrm{~mm}$ emission toward Proxima Centauri. Contours are $-3,3,6$, and 9 times $10 \mu \mathrm{Jy}_{\text {beam }}{ }^{-1}$, the rms of the image. The position of the star on 2017 April 25, measured from an optical image (error $\simeq 0^{\prime \prime} 1$ ), is marked with a + sign. The synthesized beam $\left(0\right.$. . $79 \times 0$. . $\left.69, \mathrm{PA}=50^{\circ} .3\right)$ is shown in the bottom-right corner. 
The source appears marginally elongated (deconvolved size $\sim 0$.' 6 ) in a direction with $\mathrm{PA} \simeq 130^{\circ}$.

A secondary source with a flux density of $38 \pm 10 \mu \mathrm{Jy}$ is marginally detected $(4 \sigma)$ at ICRS coordinates R.A. = $14^{\mathrm{h}} 29^{\mathrm{m}} 33^{\mathrm{s}} .604 \pm 0.011$, decl. $=-62^{\circ} 40^{\prime} 33^{\prime \prime} .88 \pm 00^{\prime \prime} 07$, at a distance of $1 . \prime 2$ and $\mathrm{PA}=114^{\circ}$ from the central star.

\section{Results and Discussion}

\subsection{The Emission from the Star}

The star (with a diameter of $\sim 0$ !'001) should appear angularly unresolved in the ALMA observations. Thus, the observed intensity peak in the ALMA $12 \mathrm{~m}$ array image (angular resolution $\simeq 0$ ! 7 ) sets an upper limit of $100 \pm 10 \mu \mathrm{Jy}$ (Figure 2) for the stellar flux density.

The expected non-thermal emission of the star at $1.3 \mathrm{~mm}$, extrapolated from the non-thermal flux density and spectral index measured by Slee et al. (2003), as well as our own ATCA measurements between 1 and $3 \mathrm{GHz}$ (J. F. Gómez et al. 2017, in preparation), is negligible $(\ll 1 \mu \mathrm{Jy})$.

The expected thermal emission from the star can be obtained from the photospheric emission model that fits the overall SED as described by Ribas et al. (2017), giving an extrapolated flux density at $1.3 \mathrm{~mm}$ of $74 \pm 4 \mu \mathrm{Jy}$. This result is fully consistent with the upper limit $(<100 \mu \mathrm{Jy})$ provided by our ALMA $12 \mathrm{~m}$ array observations (Figure 2 ), and indicates that $\sim 70 \%-80 \%$ of the flux density detected by ACA $(340 \pm 60 \mu \mathrm{Jy}$; Figure 1) does not arise from the star; therefore, we interpret it as thermal emission from circumstellar dust (see below).

\subsection{Dust around Proxima Centauri}

As shown above, the flux density of the unresolved (diameter $\lesssim 6^{\prime \prime}$ ) source detected by ACA largely exceeds what can be accounted for by the star, and we interpret the excess of $\sim 270 \mu \mathrm{Jy}$ as thermal emission from dust orbiting the star at radii $r \lesssim 3^{\prime \prime}(r \lesssim 4 \mathrm{au})$. The actual distribution of this dust can be further constrained by the ALMA $12 \mathrm{~m}$ array data.

If this emission were originated in a compact region (up to a few times the solid angle of the ALMA $12 \mathrm{~m}$ array synthesized beam), it would have been detected with a high $\mathrm{S} / \mathrm{N}$ in the ALMA image (Figure 2). However, the image only reveals two sources above the $3 \sigma$ threshold within a region of $\sim 6^{\prime \prime}$ in size (similar to the size of the ACA synthesized beam), totaling a flux density of $\sim 70 \mu \mathrm{Jy}$ after the subtraction of the estimated emission of the star. This implies that the remaining $\sim 200 \mu \mathrm{Jy}$ should be distributed over a solid angle $\gtrsim 7$ times that of the ALMA beam for its intensity to remain below the $3 \sigma$ threshold of $30 \mu \mathrm{Jy} \mathrm{beam}^{-1}$ (if a fraction of the emission was resolved out by the interferometer, this conclusion still holds). This condition requires that a significant part of the emission comes from radii larger than $\sim 1^{\prime \prime}$. Thus, with our current data we infer that there are $\sim 200 \mu \mathrm{Jy}$ of dust emission spread over a belt in a range of radii from $\sim 1^{\prime \prime}$ to $\sim 3^{\prime \prime}$, corresponding to $\sim 1.3-4$ au. A more precise determination of its distribution would require additional, more sensitive observations.

While our data are insufficient for a proper modeling, they are nevertheless enough to obtain a rough estimate of the masses involved. The mass of dust detected by ALMA can be estimated approximately as $\left(m_{\text {dust }} / M_{\oplus}\right)=0.5\left(S_{\nu} / \mathrm{Jy}\right)\left(T_{d} / \mathrm{K}\right)^{-1}$ $(d / \mathrm{pc})^{2}(\nu / 230 \mathrm{GHz})^{-2}$, where a dust opacity of $\kappa_{\nu}=$ $2 \mathrm{~cm}^{2} \mathrm{~g}^{-1}$ has been adopted (e.g., Beckwith et al. 2000). The dust temperature can be approximated as $\left(T_{d} / \mathrm{K}\right)=$
$278\left(L / L_{\odot}\right)^{0.25}(r / \mathrm{au})^{-0.5}$ (Wyatt 2008). Thus, for the dust observed at $r \simeq 1-4$ au, we obtain $T_{d} \simeq 40 \mathrm{~K}$ and a dust mass $m_{d} \simeq 4 \times 10^{-6} M_{\oplus}$.

The $1.3 \mathrm{~mm}$ continuum emission traces dust grains with $\mu \mathrm{m}$ to $\mathrm{cm}$ sizes. However, this population of small grains results from the collisional cascade involving a primordial population of larger bodies and planetesimals containing most of the mass. The equilibrium size distribution resulting from this collisional cascade can be described by a power-law of index -3.5 (Tanaka et al. 1996). Following a formulation similar to that of Wyatt \& Dent (2002), it can easily be shown that, if the primordial size distribution connects smoothly with the collisional cascade distribution, the total mass can be approximated by $m_{\text {tot }} \simeq$ $m_{\text {dust }}\left(D_{\text {max }} / D_{\text {dust }}\right)^{0.5}$, where $D_{\max } / D_{\text {dust }}$ is the ratio between the maximum sizes of the population of large bodies and that of the observed dust emission. Taking $D_{\text {dust }} \simeq 1 \mathrm{~cm}$, we obtain $m_{\text {tot }} \simeq 2200 m_{\text {dust }}\left(D_{\max } / 50 \mathrm{~km}\right)^{0.5}$. Therefore, if we integrate up to $D_{\max } \simeq 50 \mathrm{~km}$ (e.g., Greaves et al. 2004; Wyatt et al. 2007b), we obtain a total mass of $m_{\text {tot }} \simeq 10^{-2} M_{\oplus}$.

This mass is similar to that of the solar Kuiper Belt ( $\sim 10^{-2} M_{\oplus}$; Bernstein et al. 2004), which also has a similar temperature $(\sim 50 \mathrm{~K})$, but is located at a much greater distance (30-50 au) from the Sun. Given the very low luminosity of the M-dwarf star Proxima Centauri (Section 1), one would expect that physical conditions similar to those required for the solar Kuiper Belt are attained at distances much closer to the star. Therefore, we suggest that the dust emission in Proxima Centauri, arising at scales $\sim 1-4$ au, is likely tracing a Kuiper Belt analog around this star.

Additionally, we note that the central source detected by the ALMA $12 \mathrm{~m}$ array (Figure 2) appears marginally elongated along $\mathrm{PA} \simeq 130^{\circ}$, with a flux density of $106 \mu \mathrm{Jy}$ and a deconvolved size of $\sim 0.8$ au. A hint of an excess of emission in the proximity of the star and elongation along a similar PA is also observed in images made by combining the ACA and ALMA $12 \mathrm{~m}$ array data. Thus, considering the stellar emission to be $74 \mu \mathrm{Jy}$ (see Section 3.1), it might be possible that a small amount of warmer dust, with a flux density of $\sim 30 \mu \mathrm{Jy}$, is present at distances of $\sim 0.4$ au from the star. Following the same procedures as above, we estimate a characteristic temperature $T_{d} \simeq 90 \mathrm{~K}$, a dust mass of $\sim 5.5 \times 10^{-7} M_{\oplus}$, and a total mass of $\sim 10^{-3} M_{\oplus}$ for this possible hotter component.

\subsection{A Possible Outer Belt at 30 au}

The $1.3 \mathrm{~mm}$ ACA image (Figure 1) does not show direct evidence for dust structures other than the compact central source, but it shows a number of weak emission peaks that could be part of a larger structure. Since a tilted circular belt would appear as an ellipse on the sky, we performed averaging of the observed emission over elliptical annuli centered on the star in order to increase the $\mathrm{S} / \mathrm{N}$. When azimuthally averaging the intensity in annuli, the $\mathrm{S} / \mathrm{N}$ of the intensity profile at a given radius improves by a factor equal to the square root of the ratio between the length of the annulus and the beam diameter. This approach has been successfully used to infer the presence of rings and gaps in protoplanetary disks around young stars (Osorio et al. 2014; Macías et al. 2017), as well as in debris disks around old stars (Marino et al. 2017).

The radial intensity profiles obtained in this way suggest an emission peak around a radius of $\sim 23^{\prime \prime}$ ( $r \simeq 30 \mathrm{au}$ ). We analyzed a grid of different position angles and eccentricities of the ellipses. The peak appears to be better defined and stronger 


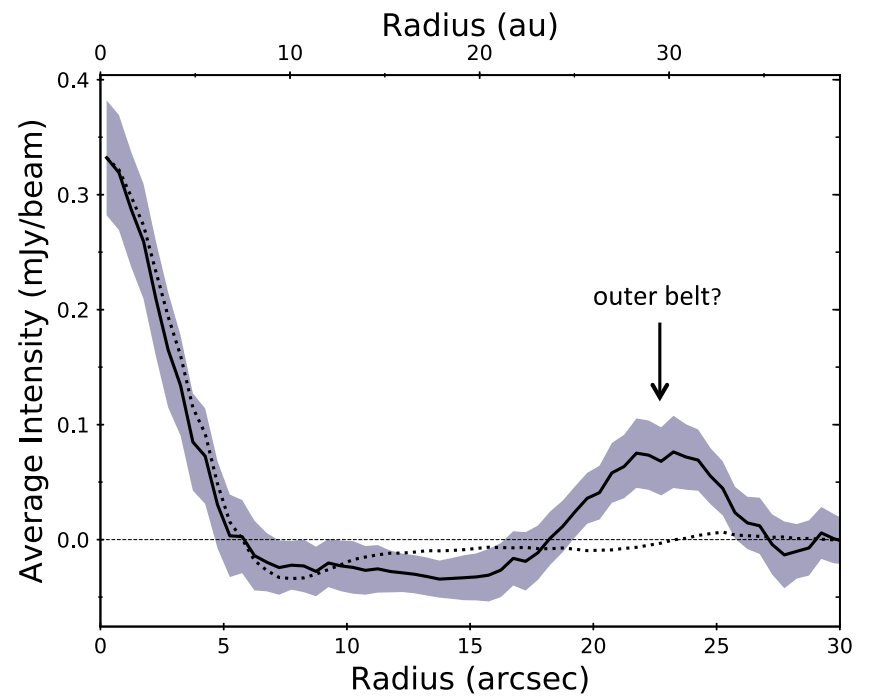

Figure 3. Azimuthally averaged radial intensity profile (solid line) of the primary-beam corrected ACA image. Averaging has been performed over elliptical annuli, centered on the position of the star and with the major axis along $\mathrm{PA}=140^{\circ}$ (see Figure 1), corresponding to the projection on the plane of the sky of circular annuli with an inclination angle $i=45^{\circ}$. The plot shows the compact central source and a possible outer belt at a (deprojected) radius of $\sim 23^{\prime \prime}$ ( $\sim 30 \mathrm{au}$ ). The gray area illustrates the $1 \sigma$ uncertainty, calculated from the standard deviation of the observed intensity within each annulus, which gives an upper limit for the uncertainty. The synthesized beam profile (dotted line) averaged over the same region is also plotted as a reference.

for a combination of the ellipse $\mathrm{PA} \simeq 140^{\circ}$ (major axis) and an eccentricity corresponding to an inclination angle $i \simeq 45^{\circ}$ (see Figures 3 and 1). Deviations from this combination of parameters produce a progressive vanishing of the feature. This feature is only detected in the ACA image because it appears at great distances from the center where the response of the ALMA $12 \mathrm{~m}$ primary beam is very low because of its smaller FWHM (see Section 2). If this belt proves to be real, it would provide a good estimate of the orientation of the orbital plane of the Proxima Centauri planetary system. If coplanarity is assumed, the true mass of the planet Proxima b (AngladaEscudé et al. 2016) would be $\sim 1.8 M_{\oplus}$. Interestingly, the PA of this outer belt is similar to that found for the elongated central source $\left(\sim 130^{\circ}\right.$; Figure 2$)$, supporting the reality of both structures and suggesting a similar orientation of the system at both small and large scales. However, we should emphasize that the detection of this outer belt is marginal, and it should be confirmed with additional observations of higher sensitivity. We note that the $45^{\circ}$ inclination of this tentative outer belt differs significantly from the $108^{\circ}$ inclination of the orbit of Proxima Centauri around Alpha Centauri AB (Kervella et al. 2017). However, we do not consider these results to be in conflict because, in general, orbital motions in a hierarchical triple system are not expected to be coplanar (e.g., Muñoz \& Lai 2015). Indeed, the orbit of the Alpha Centauri AB binary also has a significantly different inclination $\left(79^{\circ}\right.$; Kervella et al. 2016).

Thus, it is possible that Proxima Centauri is surrounded by several belts of dust (see Figure 4), one or several close to the star ( $r \lesssim 4 \mathrm{au}$ ), and another one (to be confirmed) at a large radius $(r \simeq 30 \mathrm{au})$. For this outer belt the average intensity is $\sim 76 \mu \mathrm{Jy} \mathrm{beam}^{-1}$ (Figure 3 ), and we estimate a total flux density of $\sim 1.7 \mathrm{mJy}$. The presence of such a distant belt in the very low-luminosity star Proxima Centauri is challenging. It would be extremely cold (with $T_{d} \simeq 10 \mathrm{~K}$, if the same temperature law is assumed) and its flux density would lead to a dust mass of $1.4 \times 10^{-4} M_{\oplus}$, corresponding to a total mass (including large bodies) of $0.33 M_{\oplus}$, if the same assumptions as in Section 3.2 are made, but see Krivov et al. (2013). This mass is much larger than that of the solar Kuiper Belt $\left(\sim 10^{-2} M_{\oplus}\right.$; Bernstein et al. 2004), and so far there is no known analog in the solar system. We note, however, that Herschel revealed a new class of very cold debris disks around some mature solartype stars (Eiroa et al. 2011), the origin (still unexplained) of which could share some similarities with our proposed $30 \mathrm{au}$ belt in Proxima Centauri.

\subsection{A Ring around an Undiscovered Planet at $\sim 1.6$ au?}

As noted in Section 2, our images show a marginally detected $(\sim 40 \mu \mathrm{Jy}$ at $4 \sigma)$ compact source of $1.3 \mathrm{~mm}$ emission at a projected distance of $\sim 1$ !' 2 southeast from the star (see Figure 2). This source is very intriguing. We can discard beam or cleaning artifacts, given the low beam sidelobes and high quality of the image. However, the significance of the source is marginal, and with the current data we cannot rule out the possibility that this component is just a noise peak.

If the source is real, then we can consider several possibilities. The source could be a background galaxy (Smail et al. 1997; Fujimoto et al. 2016). The probability of finding a source like this within 1 ." 2 of Proxima Centauri is small $\left(\lesssim 10^{-2}\right.$, according to the source counts of Fujimoto et al. 2016), but it cannot be completely discarded. Given the large proper motions of the star, a second-epoch observation would easily reveal whether this source moves together with the star or if it is a background static source. A substellar companion with a temperature of the order of $1000 \mathrm{~K}$ could also produce the observed emission. However, according to our calculations, such an object would produce a detectable signature in the radial velocity $(\mathrm{RV})$ of the star that has not been observed. A transient event, such as the collision between large bodies (Wyatt et al. 2007a), might produce a cloud of dust with properties similar to the observed source. However, observation of such an episodic event seems unlikely in this old star. The source might be tracing a cloud of dust orbiting in the proximity of the Lagrange points of an stillundetected planet, in a way similar to the Trojan minor planets in our solar system. However, Trojan clouds are located at the $\mathrm{L}_{4}$ and $\mathrm{L}_{5}$ Lagrange points $\sim 60^{\circ}$ ahead and behind the larger body. If the $140^{\circ}$ position angle of the $\sim 30$ au tilted belt is significant, and the inner dust disks and potential planetary orbits share the same inclination, their brightest parts would be approximately southeast and northwest, so we might be seeing the more favorably placed Trojan cloud, or possibly just the brightest part of an uneven disk that is mostly just below our detection threshold at the higher resolution.

Finally, an exciting alternative scenario is that the source traces a ring of dust surrounding an as-yet-undiscovered giant planet orbiting at a (projected) distance of $1.6 \mathrm{au}$ (orbital period $\gtrsim 75.8$ year). By analogy with the rings of Saturn we expect a power-law distribution with an index of -3.5 and a maximum particle size of $5-10 \mathrm{~m}$ (Zebker et al. 1985; Brilliantov et al. 2015), resulting in a total mass of $\sim 10^{-5} M_{\oplus}$ for such a planetary ring. Theoretical arguments (Charnoz et al. 2017) suggest that evolved planetary rings have a mass $\sim 10^{-7}$ times 


\section{Proxima Centauri}

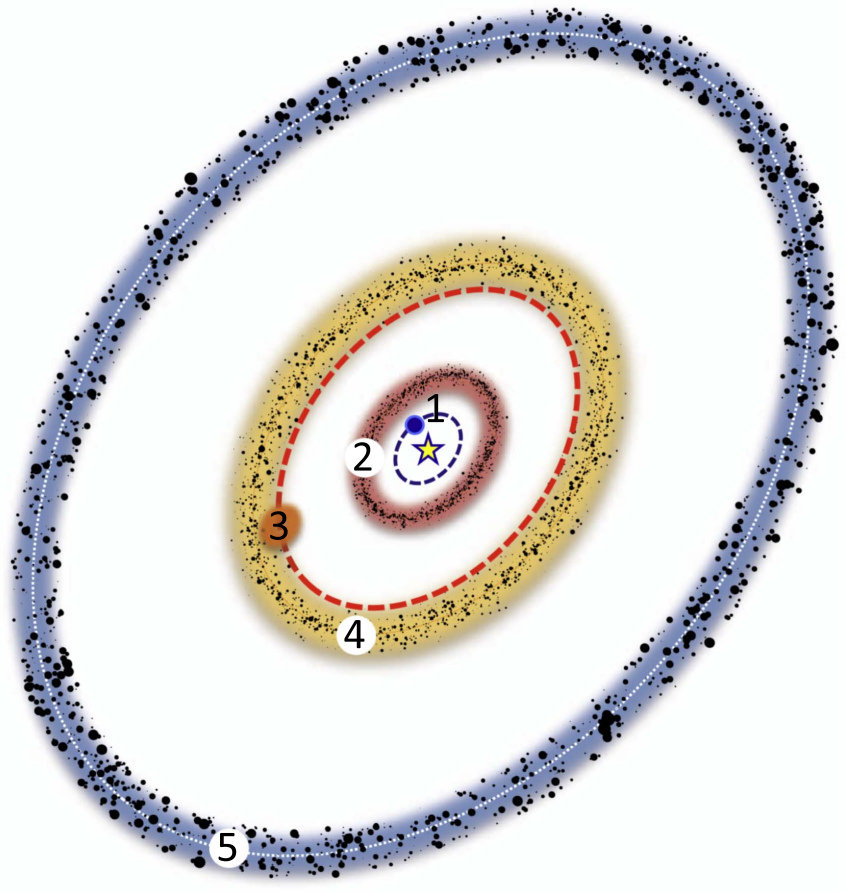

$\begin{array}{lr}\text { 1. Proxima b planet } & r=0.05 \mathrm{au} \\ \text { 2. Warm dust? } & r \approx 0.4 \mathrm{au} \\ \text { 3. Unknown source? } & r=1.6 \mathrm{au} \\ \text { 4. Cold belt } & r \approx 1-4 \mathrm{au} \\ \text { 5. Outer belt? } & r=30 \mathrm{au}\end{array}$

Figure 4. Sketch (not to scale) of the proposed components in the Proxima Centauri planetary system. Question marks indicate marginally detected features.

the mass of the planet. Thus, under this scenario, we would expect a planet of mass $\sim 100 M_{\oplus}$, the mass of Saturn, to account for the observed $1.3 \mathrm{~mm}$ emission. There is no clear RV signal to indicate that such a planet is present in the data of the long-term monitoring of the star. Further observations are being undertaken to confirm or rule out this intriguing possibility. At any rate, our study shows that ALMA provides already the necessary sensitivity and resolution to detect rings around exoplanets in Alpha Centauri, and perhaps in other nearby stars.

This paper makes use of the following ALMA data: ADS/JAO. ALMA\#2016.A.00013.S. ALMA is a partnership of ESO (representing its member states), NSF (USA) and NINS (Japan), together with NRC (Canada) and NSC and ASIAA (Taiwan) and KASI (Republic of Korea), in cooperation with the Republic of Chile. The Joint ALMA Observatory is operated by ESO, AUI/ NRAO and NAOJ. This work has been partially supported by MINECO (Spain) grants AYA-2014-56637-C2-1-P, AYA201457369-C3-3-P, AYA2015-63939-C2-1-P, AYA2016-79425-C3-3P, AYA2016-80889-P, ESP2014-54032-R, ESP2014-54362-P, and ESP2016-76076-R, co-funded with FEDER funds. I.J.-S. acknowledges financial support from the STFC through an Ernest Rutherford Fellowship (proposal number ST/L004801). J.S.J. acknowledges support by Fondecyt grant 1161218 and partial support by CATA-Basal (PB06, CONICYT). Z.M.B. acknowledges partial support from ALMA-CONICYT FUND. We are deeply indebted to the ALMA Observatory staff for their efficient handling of the observations and specially to the UK ARC Node, and data analysts in North American and European ALMA Regional Centers for their fast reaction in the data reduction process.

\section{ORCID iDs}

Guillem Anglada (1) https://orcid.org/0000-0002-7506-5429 Pedro J. Amado (1) https://orcid.org/0000-0002-8388-6040 Jose L. Ortiz (i) https://orcid.org/0000-0002-8690-2413 José F. Gómez (i) https://orcid.org/0000-0002-7065-542X Enrique Macías (i) https://orcid.org/0000-0003-1283-6262 José L. Gómez i https://orcid.org/0000-0003-4190-7613 Miguel A. Pérez-Torres (ib https://orcid.org/0000-00015654-0266

Guillem Anglada-Escudé i https://orcid.org/0000-00023645-5977

James S. Jenkins (1) https://orcid.org/0000-0003-2733-8725 Izaskun Jimenez-Serra (1) https://orcid.org/0000-00034493-8714

Manuel López-Puertas (ii) https://orcid.org/0000-00032941-7734

Ignasi Ribas (1) https://orcid.org/0000-0002-6689-0312 Cristina Rodríguez-López (i) https://orcid.org/0000-0001$5559-7850$

\section{References}

Absil, O., Defrère, D., Coudé du Foresto, V., et al. 2013, A\&A, 555, A104 Anglada-Escudé, G., Amado, P. J., Barnes, J., et al. 2016, Natur, 536, 437 Bazot, M., Christensen-Dalsgaard, J., Gizon, L., \& Benomar, O. 2016, MNRAS, 460, 1254

Beckwith, S. V. W., Henning, T., \& Nakagawa, Y. 2000, in Protostars and Planets IV, ed. V. Mannings, A. P. Boss, \& S. S. Russell (Tucson, AZ: Univ. of Arizona Press), 533

Bernstein, G. M., Trilling, D. E., Allen, R. L., et al. 2004, AJ, 128, 1364

Brilliantov, N., Krapivsky, P. L., Bodrova, A., et al. 2015, PNAS, 112, 9536

Charnoz, S., Canup, R. M., Crida, A., \& Dones, L. 2017, in Planetary Ring Systems, ed. M. S. Tiscareno \& C. D. Murray (Cambridge: Cambridge Univ. Press), in press (arXiv:1703.09741)

Chavez-Dagostino, M., Bertone, E., Cruz-Saenz de Miera, F., et al. 2016, MNRAS, 462, 2285

di Folco, E., Absil, O., Augereau, J.-C., et al. 2007, A\&A, 475, 243

Eiroa, C., Marshall, J. P., Mora, A., et al. 2011, A\&A, 536, L4

Fujimoto, S., Ouchi, M., Ono, Y., et al. 2016, ApJS, 222, 1

Greaves, J. S., Wyatt, M. C., Holland, W. S., \& Dent, W. R. F. 2004, MNRAS, 351, L54

Kervella, P., Mignard, F., Mérand, A., \& Thévenin, F. 2016, A\&A, 594, A107

Kervella, P., Thévenin, F., \& Lovis, C. 2017, A\&A, 598, L7

Krivov, A. V., Eiroa, C., Löhne, T., et al. 2013, ApJ, 772, 32

Lestrade, J.-F., Matthews, B. C., Sibthorpe, B., et al. 2012, A\&A, 548, A86

Lurie, J. C., Henry, T. J., Jao, W.-C., et al. 2014, AJ, 148, 91

MacGregor, M. A., Lawler, S. M., Wilner, D. J., et al. 2016, ApJ, 828, 113

Macías, E., Anglada, G., Osorio, M., et al. 2017, ApJ, 838, 97

Marino, S., Wyatt, M. C., Panić, O., et al. 2017, MNRAS, 465, 2595

Matthews, B. C., Krivov, A. V., Wyatt, M. C., Bryden, G., \& Eiroa, C. 2014, in Protostars and Planets VI, ed. H. Beuther et al. (Tucson, AZ: Univ. of Arizona Press), 521

Morbidelli, A., Levison, H. F., Tsiganis, K., \& Gomes, R. 2005, Natur, 435, 462 Muñoz, D. J., \& Lai, D. 2015, PNAS, 112, 9264

Osorio, M., Anglada, G., Carrasco-González, C., et al. 2014, ApJL, 791, L36 Raymond, S. N., Armitage, P. J., Moro-Martín, A., et al. 2011, A\&A, 530, A62 Ribas, I., Gregg, M. D., Boyajian, T. S., \& Bolmont, E. 2017, A\&A, 603, A58 Slee, O. B., Willes, A. J., \& Robinson, R. D. 2003, PASA, 20, 257

Smail, I., Ivison, R. J., \& Blain, A. W. 1997, ApJL, 490, L5

Tanaka, H., Inaba, S., \& Nakazawa, K. 1996, Icar, 123, 450

van Leeuwen, F. 2007, A\&A, 474, 653

Wyatt, M. C. 2008, ARA\&A, 46, 339

Wyatt, M. C., \& Dent, W. R. F. 2002, MNRAS, 334, 589

Wyatt, M. C., Smith, R., Greaves, J. S., et al. 2007a, ApJ, 658, 569

Wyatt, M. C., Smith, R., Su, K. Y. L., et al. 2007b, ApJ, 663, 365

Zebker, H. A., Marouf, E. A., \& Tyler, G. L. 1985, Icar, 64, 531 